\title{
Thesis
}

\section{A modular classifier concept for activity recognition on mobile phones}

\author{
Martin Berchtold \\ TECO, Karlsruhe Institute of Technology (KIT), Germany
}

Keywords: Modular activity recognition, flexibility, extensibility, robustness, resources, conditionality

On October 26, 2011, Martin Berchtold defended his Ph.D. dissertation titled: "A Modular Classifier Concept for Activity Recognition on Mobile Phones". Supervisors where Lars Wolf, TU Braunschweig and Michael Beigl, TECO, KIT. Martin Berchtold finished his diploma in 2007 with the diploma thesis "Quality Management of Contexts - Reliability with Fuzzy Logic". Afterwards he attended the TECO research lab of the University of Karlsuhe as research assistant. His early work concerned the bulk reading of RFID labels at item level tagging in retail stores (German research project "LoCostix") and the development of an infrastructure-less navigation system for firefighters (German research project "Landmarke"). In 2009 Martin Berchtold changed to the research group of Prof. Beigl at the University of Braunschweig where he worked on the topic of activity recognition on smart phones in the project "LocCom" ("IT-Ecosystems" joint project of the state of
Niedersachsen). In 2010 he joined Prof. Beigl when he accepted a call to the KIT, but partly he continued his work on "LocCom". His main research focus was on reliability measures, classifiers and machine learning for activity recognition with accelerometer data which is also the topic of his $\mathrm{PhD}$ thesis.

\section{Thesis summary}

In his thesis a novel modular activity recognition using accelerometer sensors on mobile phones is presented, which includes solutions to five challenges of activity recognition on mobile phones: flexibility, extensibility, robustness, resource efficiency and awareness for different conditionalities. The novel modularity of the classification process enables the individual adaption of parts of the activity recognition to offer flexibility. The novel modular recogni-

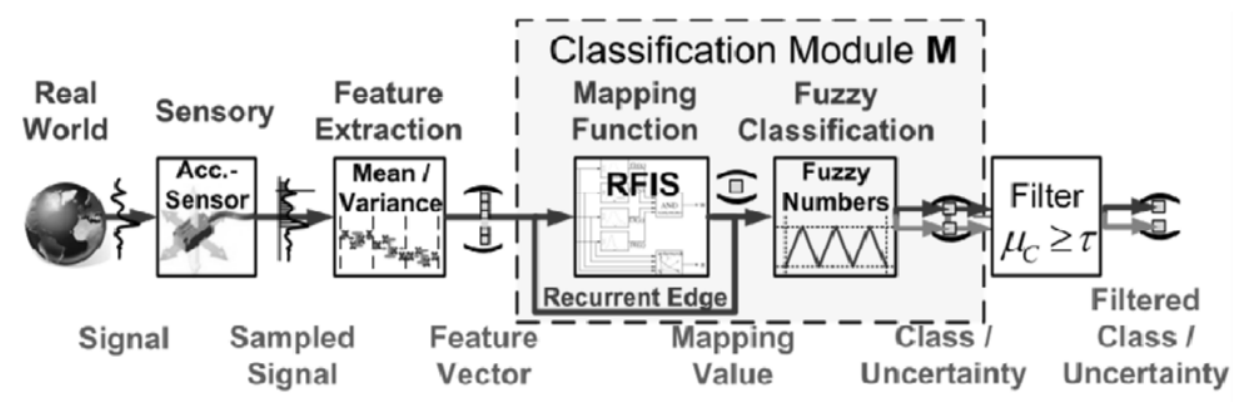

Fig. 1. Classification architecture. 
tion is extensible by new modules which detect new activities. A novel recurrent classification process stabilizes the recognition and enables the derivation of a reliability measure, which in conjunction result in a robust activity recognition. Only one module and not the whole activity recognition is active at each point in time, which decreases the calculation effort and therefore the energy consumption, which saves resources in general. Each module of the activity recognition can be suited for dealing with one conditionality, through which neither the complexity of the recognition is increased nor the accuracy is significantly lowered. All these solutions to the challenges of activity recognition on mobile phones are rounded by a service called ActiServ, which supports the novel system on the common user's phone. 